\title{
Modelagem do crescimento de cultivares de cana-de-açúcar no período de formação da cultura
}

\author{
Evandro L. da S. Batista', Sérgio Zolnier', Aristides Ribeiro', \\ Gustavo B. Lyra ${ }^{2}$, Thieres G. F. da Silva ${ }^{3}$ \& Davi Boehringer ${ }^{1}$
}

\begin{abstract}
RESUMO
Este estudo teve como objetivo ajustar modelos de crescimento ao acúmulo de matéria seca da parte aérea da cana-de-açúcar durante o período de formação da cultura, para as cultivares RB92579, RB867515, RB928064 e RB855453. Dados de matéria seca foram obtidos durante a execução de experimento em condições de campo entre 26/08/11 e 18/05/12. Os dados experimentais foram coletados a partir dos 45 dias após o plantio, em intervalos de uma ou duas semanas, totalizando 17 datas de amostragem. Os valores obtidos foram submetidos à análise de regressão não linear para determinação dos parâmetros dos modelos expolinear, logístico e Gompertz. Todos os modelos avaliados foram capazes de simular adequadamente o acúmulo de matéria seca pelas cultivares ao longo do período estudado, com valores de coeficiente de determinação ajustados $\left(R^{2}{ }_{\text {aj }}\right)$ acima de 0,9245 . Com base no modelo de crescimento expolinear, constatouse que, ao final do período experimental, a massa seca foi ligeiramente maior para a cultivar RB855453 em comparação à RB867515, as quais se destacaram em relação às cultivares RB928064 e RB92579.
\end{abstract}

Palavras-chave: Saccharum officinarum, modelos de crescimento, análise de crescimento

\section{Modeling of growth of sugarcane cultivars during the crop formation period}

\begin{abstract}
The objective of the present study was to adjust growth models to the above-ground accumulated sugarcane dry matter, during the crop formation period, for cultivars RB92579, RB867515, RB928064 and RB855453. Dry matter data were obtained during the execution of experiment under field conditions from October 26, 2011 to May 18, 2012. Experimental data were collected from 45 days after planting, in one or two week time intervals, totalizing 17 sampling dates. The obtained values were subjected to nonlinear regression analysis in order to determine the parameters of the expolinear, logistic and Gompertz models. All evaluated models were capable of adequately simulating the dry matter accumulation by the cultivars during the studied period, with values of the adjusted determination coefficient above 0.9245 . Based on the expolinear growth model, it was observed, at the end of the experimental period, that the accumulated dry mass was stightly superior for the cultivar RB855453 as compared to the RB867515, which outperformed the cultivars RB928064 and RB92579.
\end{abstract}

Key words: Saccharum officinarum, growth models, growth analysis

'DEA/UFV, Av. P. H. Holfs, s/n, CEP 36570-000, Viçosa, MG. Fone: (31) 3899-1859. E-mail: batista@ufv.br; zolnier@ufv.br; ribeiro@ufv.br; davib82@hotmail.com

${ }^{2}$ DCA/UFRRJ, Rodovia BR 465, km 07, CEP 23890-000, Seropédica, RJ. Fone: (21) 3787-4033. E-mail: gblyra@gmail.com

${ }^{3}$ UAST/UFRPE, Fazenda Saco, Zona Rural, s/n, CEP 56900-000, Serra Talhada, PE. Fone: (87) 3831-1927. E-mail: thieres@uast.ufrpe.br 


\section{INTRODUÇÃO}

A cultura da cana-de-açúcar é de grande importância para a economia brasileira, uma vez que o Brasil, além de ser o maior produtor mundial, se destaca na produção de açúcar e etanol (MAPA, 2012). Na última década, houve incremento considerável na área plantada e na produção, em virtude do lançamento de cultivares mais produtivas e da implantação de novas unidades industriais, estimulados pelo aumento das exportações e pela crescente participação dos veículos bicombustíveis na frota brasileira (Sousa \& Macedo, 2010).

Estima-se que a área cultivada com cana-de-açúcar, para a atividade sucroalcooleira na safra de 2012/2013, será de 8,53 milhões de hectares, proporcionando uma produção estimada de 596,6 milhões de toneladas, que representam um aumento de $6,5 \%$ em relação à safra 2011/2012 (CONAB, 2012). A atual demanda por produtos derivados da cana-de-açúcar tem incentivado a realização de pesquisas que buscam o entendimento do crescimento e desenvolvimento das plantas, em decorrência de alterações de práticas agronômicas e da variação dos elementos meteorológicos durante o ciclo da cultura buscando, assim, o uso eficiente dos recursos ambientais disponíveis.

Um manejo adequado pode ser obtido por meio da identificação dos padrões de crescimento, que possibilitam selecionar cultivares adaptadas aos diferentes ambientes de produção e estimar o período mais apropriado para o plantio. Neste sentido, a análise de crescimento é uma ferramenta extremamente útil, pois permite identificar diferenças morfológicas e quantificar a produção líquida resultante do processo fotossintético, facilitando a compreensão das interações ambiente-planta (Benincasa, 1988). Esta análise pode ser aprimorada por meio de modelos empíricos, semiempíricos ou mecanísticos, os quais possibilitam estimar o acúmulo de matéria seca em algumas fases do período de crescimento ou ao longo do ciclo da cultura.

Embora os modelos mecanísticos tenham uma formulação científica, funções empíricas, como a logística e a proposta por Gompertz (Amer \& Williams, 1957; Richards, 1959) têm sido usadas para modelar o crescimento das plantas, o acúmulo de matéria seca e a produtividade (Lyra et al., 2008; Karadavut et al., 2010; Sepaskhah et al., 2011). Outro modelo muito utilizado para estimativa do crescimento é a função expolinear proposta por Goudriaan \& Monteith (1990). Considerada semiempírica, baseia-se, embora indiretamente, na existência de uma relação exponencial no início do ciclo e, posteriormente, linear, entre o acúmulo de massa seca total e a quantidade de radiação fotossinteticamente ativa interceptada pelo dossel.

A grande vantagem dos modelos matemáticos mencionados, em relação a outros modelos empíricos não lineares, como a função polinomial, é que seus parâmetros permitem interpretação biológica, possibilitando, desta forma, inferir sobre alterações do ambiente, práticas agrícolas e épocas de plantio, entre outros fatores que influenciam o crescimento e o desenvolvimento das plantas.

Na cultura da cana-de-açúcar, exemplos da utilização de modelos de crescimento incluem estudos sobre o efeito de doses de fertilizantes na produtividade (Gava et al., 2001; Santos et al., 2009); desempenho produtivo de cultivares em condições ambientais particulares (Oliveira et al., 2004; Silva et al., 2012), eficiência do uso da água de cultivares (Oliveira et al., 2010) e evolução temporal de variáveis agronômicas (Simões et al., 2005).

Durante seu crescimento, a cana-de-açúcar passa por quatro estádios fenológicos distintos, denominados germinação, perfilhamento, crescimento intenso e maturação (Gascho \& Shih, 1983). O tempo transcorrido durante os estádios de perfilhamento e de crescimento intenso é conhecido como período de formação da cana-de-açúcar. Estima-se que cerca de 70 a $80 \%$ do rendimento final da cultura são determinados pelo acúmulo de matéria seca neste período (Almeida et al., 2008). Desta forma, o ajuste de modelos aos dados de matéria seca, durante o período de formação da cana-de-açúcar, subsidia informações para a tomada de decisão na seleção de cultivares mais adaptadas a um sistema de cultivo específico.

O tempo transcorrido após o plantio tem sido usado como variável independente em vários modelos de crescimento. Todavia, devido à importância da relação entre temperatura do ambiente de cultivo e o acúmulo de biomassa, a variável graus-dia acumulados tem mostrado resultados melhores para estimativa do crescimento em ambientes diferentes dos utilizados para estimativa dos parâmetros dos modelos (Almeida et al., 2008; Lyra et al., 2008).

Objetivou-se, com este trabalho, avaliar o ajuste dos modelos expolinear, logístico e Gompertz ao acúmulo de matéria seca da parte aérea de quatro cultivares de cana-deaçúcar durante o período de formação da cultura, tendo grausdia acumulados após o plantio como variável independente desses modelos matemáticos.

\section{Material e Métodos}

Este trabalho foi conduzido no período de 28/10/2011 a 18/05/2012 em uma área experimental destinada a pesquisas com a cultura de cana-de-açúcar. De acordo com a classificação de Köppen, o clima da região é do tipo Cwa, caracterizado como temperado quente - mesotérmico, com chuvas no verão e seca no inverno, sendo que a temperatura média no mês mais quente é superior a $22{ }^{\circ} \mathrm{C}$.

Dados de temperatura e umidade relativa do ar foram medidos com um sensor conjugado (modelo HMP60, Vaisala, Woburn, MA, USA), que foi instalado em um mastro localizado próximo à área de cultivo. Neste mesmo mastro, foi instalado um piranômetro (modelo LI-200SA, Li-Cor Inc., Lincoln, NE, USA) para medição da radiação solar global. A conversão analógico-digital dos sinais dos sensores foi efetuada por meio de um sistema automático de aquisição de dados, composto por um módulo analógico (modelo LR-7018, LR Informática Industrial, Porto Alegre, RS) e outro conversor de sinais digitais (modelo LR-7520, LR Informática Industrial, Porto Alegre, RS) que permite a comunicação entre os protocolos RS-485 e RS-232, utilizados para transferência de dados a longa e curta distâncias, respectivamente. Os dados ambientais medidos foram armazenados em intervalos de um minuto, totalizando 1440 medições por dia. Os dados de precipitação e velocidade do ar foram obtidos a partir da estação meteorológica do INMET. 
Para o preparo do solo, foram realizadas duas gradagens seguidas do sulcamento do terreno. A calagem e a adubação foram efetuadas com base na análise físico-química do solo, realizada a partir de amostras coletadas entre 0 e $20 \mathrm{~cm}$ de profundidade (Tabela 1).

Tabela 1. Caracterização química e física do solo da área experimental

\begin{tabular}{lccc}
\hline \multicolumn{1}{c}{$\begin{array}{c}\text { Componente } \\
\text { analisado }\end{array}$} & Unidades & Resultados* & Interpretação** \\
$\mathrm{pH} \mathrm{H} \mathrm{H}_{2} \mathrm{O}(1: 2,5)$ & - & 5,39 & Baixo \\
$\mathrm{P}$ disponivel & $\mathrm{mg} \mathrm{dm}^{-3}$ & 5,00 & Baixo \\
$\mathrm{K}$ & $\mathrm{mg} \mathrm{dm}^{-3}$ & 50,00 & Médio \\
$\mathrm{Ca}$ & $\mathrm{Cmol}_{\mathrm{c}} \mathrm{dm}^{-3}$ & 2,70 & Bom \\
$\mathrm{Mg}$ & $\mathrm{Cmol}_{\mathrm{c}} \mathrm{dm}^{-3}$ & 0,65 & Médio \\
$\mathrm{Al}$ & $\mathrm{Cmol}_{\mathrm{c}} \mathrm{dm}^{-3}$ & 0,00 & Muito baixo \\
$\mathrm{H}+\mathrm{Al}$ & $\mathrm{Cmol}_{\mathrm{c}} \mathrm{dm}^{-3}$ & 4,00 & Médio \\
Soma de bases & $\mathrm{Cmol}_{\mathrm{c}} \mathrm{dm}^{-3}$ & 3,48 & Médio \\
$\mathrm{CTC}$ efetiva & $\mathrm{Cmol}_{\mathrm{c}} \mathrm{dm}^{-3}$ & 3,48 & Médio \\
$\mathrm{CTC}$ a pH 7 & $\mathrm{Cmol}_{\mathrm{c}} \mathrm{dm}^{-3}$ & 7,48 & Médio \\
V & $\%$ & 46,50 & Médio \\
Areiagrossa & $\%$ & 20,00 & \\
Areiafina & $\%$ & 11,00 & \\
Silte & $\%$ & 17,00 & \\
Argila & $\%$ & 52,00 & \\
Classe textural & & & Argila \\
\hline
\end{tabular}

*Análise realizada para amostras retiradas da camada de $0-20 \mathrm{~cm}$; ${ }^{\star \star}$ Interpretação proposta por Ribeiro et al. (1999)

A adubação de plantio, constituída de $45 \mathrm{~kg} \mathrm{ha}^{-1}$ de $\mathrm{N}$, $120 \mathrm{~kg} \mathrm{ha}^{-1}$ de $\mathrm{P}_{2} \mathrm{O}_{5}$ e $45 \mathrm{~kg} \mathrm{ha}^{-1}$ de $\mathrm{K}_{2} \mathrm{O}$, foi aplicada no fundo do sulco. Aos 60 dias após o plantio (DAP), foi feita uma adubação de cobertura, com a utilização de $45 \mathrm{~kg} \mathrm{ha}^{-1}$ de $\mathrm{N}$ e $45 \mathrm{~kg} \mathrm{ha}^{-1}$ de $\mathrm{K}_{2} \mathrm{O}$ nas linhas das plantas, seguida de incorporação ao solo. $\mathrm{O}$ sistema de plantio adotado foi o manual, cujos colmos foram distribuídos de forma contínua nos sulcos que tinham $30 \mathrm{~cm}$ de profundidade; ao longo de cada sulco, foram dispostos dois colmos paralelamente na posição pé com ponta e, posteriormente, cortados em toletes com 3 a 4 gemas, totalizando 18 gemas $\mathrm{m}^{-1}$; em seguida, os toletes foram cobertos com uma camada de 5 a $10 \mathrm{~cm}$ de solo.

As parcelas experimentais foram compostas por 7 linhas de $30 \mathrm{~m}$, com espaçamento de $1,40 \mathrm{~m}$ entre linhas. $\mathrm{O}$ experimento foi analisado em delineamento completamente casualizado com 5 repetições, sendo que os tratamentos foram constituídos de quatro cultivares (RB92579, RB867515, RB928064 e RB855453). Com o objetivo de atender à demanda hídrica dessas cultivares, foi acionado um sistema de irrigação por aspersão convencional, o qual foi utilizado apenas duas vezes durante o período de cultivo.

Para obtenção dos dados de biomassa seca da parte aérea foram amostradas cinco plantas de cada cultivar em intervalos de uma ou duas semanas, dos 45 aos 205 dias após o plantio, totalizando 17 datas de avaliação. Considerou-se $0,5 \mathrm{~m}$ iniciais da área de plantio como bordadura e, a partir daí, foram realizadas amostragens representativas em $1 \mathrm{~m}$ linear desta área de plantio para cada avaliação.

Os perfilhos foram amostrados ao nível do solo e levados para o laboratório onde foram divididos em folhas e colmos. Para obtenção da matéria seca das partes da planta, as amostras foram levadas para uma estufa com circulação de ar, onde permaneceram na temperatura de $70{ }^{\circ} \mathrm{C}$ até atingir peso constante; em seguida, a matéria seca total da parte aérea das plantas foi obtida pela soma da matéria seca das folhas e colmos.

De posse dos resultados de matéria seca da parte aérea acumulada ao longo do período experimental, foram ajustados os parâmetros dos modelos expolinear, logístico e Gompertz, em função dos graus-dias acumulados após o plantio, utilizando-se os seguintes modelos matemáticos:

- Expolinear:

$$
\ln (\mathrm{MS})=\ln \left(\mathrm{c}_{\mathrm{m}} / \mathrm{r}\right)+\ln \left\{\ln \left[1+\exp \left(\mathrm{r}\left(\mathrm{GD}-\mathrm{t}_{\mathrm{b}}\right)\right)\right]\right\}
$$

- Logístico:

$$
\ln (\mathrm{MS})=\ln \left(\mathrm{MS}_{\mathrm{f}}\right)-\ln \left[1+\left(\mathrm{MS}_{\mathrm{f}} / \mathrm{MS}_{0}-1\right) \exp (-\mathrm{r} \mathrm{GD})\right]
$$

- Gompertz:

$$
\ln (\mathrm{MS})=\ln \left(\mathrm{MS}_{0}\right)+\mathrm{r}\left[1-\exp \left(-\mathrm{r}_{\mathrm{d}} \mathrm{GD}\right)\right] / \mathrm{r}_{\mathrm{d}}
$$

em que:

GD - graus-dia acumulados após o plantio, ${ }^{\circ} \mathrm{C}$

$\mathrm{MS}_{0}$ - matéria seca inicial da parte aérea, $\mathrm{g}$ planta ${ }^{-1}$

$\mathrm{MS}_{\mathrm{f}}$ - matéria seca final da parte aérea, g planta ${ }^{-1}$

$\mathrm{r} \quad$ - taxa máxima de crescimento relativo, $\mathrm{g} \mathrm{g}^{-1}{ }^{\circ} \mathrm{C}^{-1}$

$\mathrm{c}_{\mathrm{m}} \quad$ - taxa máxima de crescimento absoluto, g planta ${ }^{-1} \mathrm{C}^{-1}$

$r_{d}$ - parâmetro que define o decréscimo da taxa de crescimento relativo, adimensional

$t_{b} \quad$ - tempo perdido ou tempo necessário para o fechamento do dossel, ${ }^{\circ} \mathrm{C}$

Os graus-dia acumulados após o plantio foram determinados pela Eq. 4, considerando-se a temperatura base de $10{ }^{\circ} \mathrm{C}$, de acordo com Smit \& Singels (2006).

$$
\mathrm{GD}=\sum_{\mathrm{i}=1}^{\mathrm{n}}\left(\mathrm{T}_{\mathrm{m}}-\mathrm{T}_{\mathrm{b}}\right)
$$

em que:

$\mathrm{T}_{\mathrm{m}} \quad$ - temperatura média diária do $a$,,${ }^{\circ} \mathrm{C}$

$\mathrm{T}_{\mathrm{b}}^{\mathrm{m}} \quad$ - temperatura base da cultura, ${ }^{\circ} \mathrm{C}$.

\section{Resultados E Discussão}

Na Figura 1 são apresentados os valores observados, durante o período experimental, dos seguintes elementos meteorológicos: a) radiação solar global $\left(R_{g}\right)$ e déficit de pressão de saturação de vapor d'água no ar $\left(\mathrm{DPV}_{\mathrm{ar}}{ }_{\mathrm{g}}\right)$ (Figura 1A); b) velocidade do ar (u) (Figura 1B); c) temperatura $\left(\mathrm{t}_{\mathrm{ar}}\right)$ e umidade relativa do ar $\left(\mathrm{UR}_{\mathrm{ar}}\right)$ (Figura 1C) e d) precipitação e irrigação (Figura 1D). No caso da radiação solar global, os valores apresentados se referem à integração das medidas instantâneas ao longo do período diurno. Similarmente, os valores de precipitação e de irrigação correspondem aos totais diários, enquanto as demais variáveis se referem às médias diárias observadas a partir de medidas realizadas em intervalos de $1 \mathrm{~min}$. 
Neste período, a temperatura do ar média diária foi de 21,2 ${ }^{\circ} \mathrm{C}$, com valores extremos de 15,6 e $24,1{ }^{\circ} \mathrm{C}$ (Figura 1C). Esta amplitude caracterizou condições térmicas abaixo da faixa considerada ótima para o crescimento da cana-de-açúcar que, segundo Almeida et al. (2008), situa-se entre 25 e $33^{\circ} \mathrm{C}$. As maiores temperaturas ocorreram entre meados de janeiro a março, com valores médios de $22,4{ }^{\circ} \mathrm{C}$ que contribuíram para que o $\mathrm{DPV}_{\text {ar }}$ atingisse valores de 7,24 $\mathrm{hPa}$ (Figura 1A). Os menores valores de $\mathrm{DPV}_{\text {ar }}$ foram observados nos meses de dezembro a janeiro, quando foi constatado o maior número de eventos de chuvas.

Verificou-se que, a partir de abril, ocorreu redução nos valores de $R_{g}$ com a transição da estação do outono para o inverno, o que ocasionou diminuição da temperatura do ar. Os valores observados máximo e mínimo de $\mathrm{R}_{\mathrm{g}}$ no período experimental foram, respectivamente, $27 \mathrm{MJ} \mathrm{m}^{-2} \mathrm{~d}^{-1}(11 / 11 / 2011)$ e 2,21 MJ m ${ }^{-2} \mathrm{~d}^{-1}(14 / 05 / 2012)$.

No que se refere à velocidade do vento, os maiores valores ocorreram nos primeiros meses do presente estudo, cujo valor máximo foi $2,83 \mathrm{~m} \mathrm{~s}^{-1}$ (26/10/2011). Em contraste, o menor valor foi observado no dia 10/01/2012, sendo de $0,13 \mathrm{~m} \mathrm{~s}^{-1}$ (Figura 1B).

A precipitação pluvial, totalizada ao longo do período experimental, foi de $1.334 \mathrm{~mm}$. O valor total diário máximo de chuva foi de $89,8 \mathrm{~mm}(01 / 01 / 2012)$. Para suprir a redução da precipitação no final de fevereiro e início do mês de março, um sistema de irrigação foi acionado nos dias 27/02 e 11/03 quando se aplicou uma lâmina de irrigação de $35 \mathrm{~mm}$ em cada dia (Figura 1D).

A.

DAP

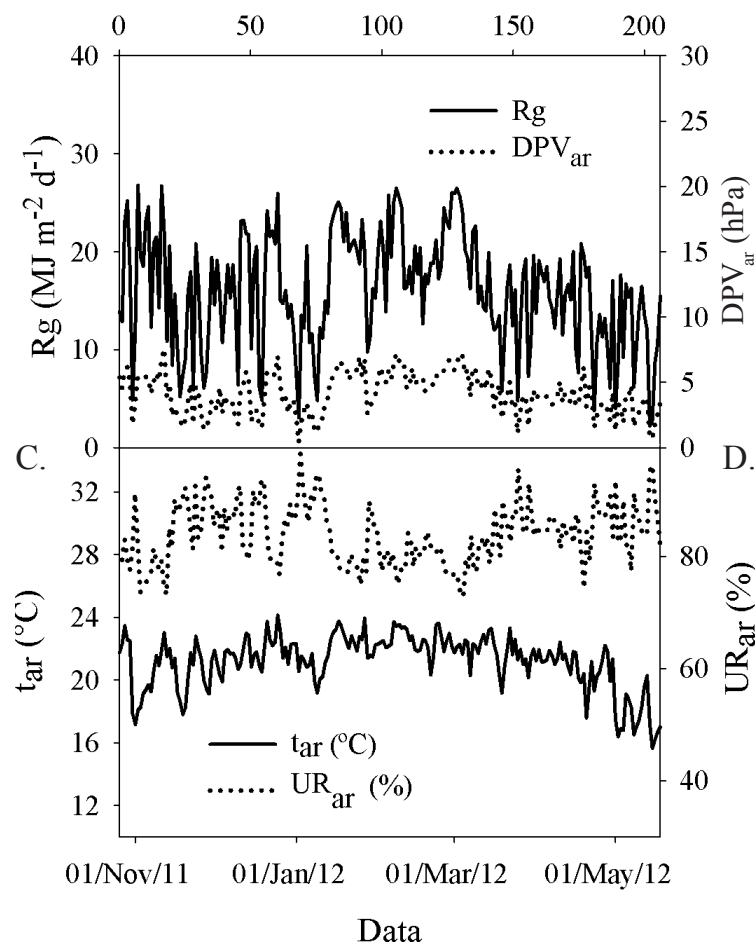

O crescimento dos cultivares de cana-de-açúcar, durante o período de formação da cultura, foi condicionado pelos valores dos elementos meteorológicos especificados anteriormente. Por meio da análise de regressão dos dados de massa seca da parte aérea, verificou-se que os modelos estudados apresentaram ótimo desempenho, com valores elevados para o coeficiente de determinação ajustado $\left(\mathrm{R}_{\text {aj }}^{2}\right)$, sendo superiores a 0,92 para as quatro cultivares. Este fato sinaliza que os modelos conseguiram explicar a maior parte da variação do acúmulo de massa seca (Figuras 2 e 3).

Os maiores valores de $\mathrm{R}_{\text {aj }}{ }_{\text {ja }}$ foram proporcionados pelo modelo expolinear, estando compreendidos entre 0,9326 (RB928064) e 0,9476 (RB855453). Tais resultados estão próximos dos observados por Costa et al. (1999) ao ajustarem este modelo aos dados de matéria seca da soja, quando foram encontrados valores de $\mathrm{R}^{2}$ da ordem de 0,98 . Confalone et al. (2010) avaliaram a adequação deste modelo para simular o crescimento de cultivares de soja submetidas ao déficit hídrico e relataram simulações satisfatórias da variação do crescimento com valores de $\mathrm{R}^{2}$ próximos de 0,99 . Os autores ressaltam ainda a capacidade do modelo para descrever o crescimento da cultura em condições ambientais distintas.

Com exceção da cultivar RB928064, os valores de $\mathrm{R}_{\text {aij, }}^{2}$ correspondentes ao modelo logístico, foram da ordem de 0,94 (Figuras 2 e 3, letras C e D). Santos et al. (2009) obtiveram valores de coeficiente de determinação da ordem de 0,99 , quando utilizaram este modelo em simulações do acúmulo de matéria fresca da cana-de-açúcar submetida a diferentes fontes de fósforo. Em estudo realizado com a cultura do milho, Lyra
B.

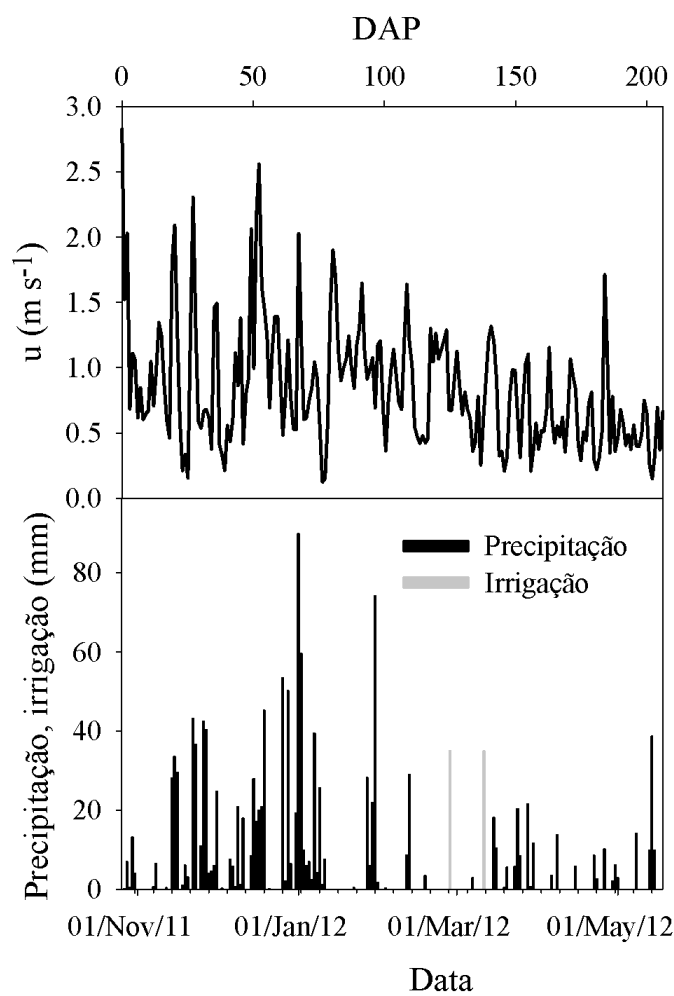

Figura 1. Variação dos elementos meteorológicos ao longo do período de formação da cana-de-açúcar, no ciclo de cana-planta. As medidas se referem aos totais diários de radiação solar global $\left(\mathrm{R}_{\mathrm{g}}\right)$ e valores médios diários do déficit de pressão de saturação de vapor d'água no vento $\left(D P V_{a r}\right)(A)$; velocidade do vento $(u)(B)$; temperatura $\left(t_{a r}\right)$ e umidade relativa do $\operatorname{ar}\left(U R_{\mathrm{ar}}\right)(\mathrm{C})$. Adicionalmente, são apresentados os totais diários de precipitação e irrigação (D) 


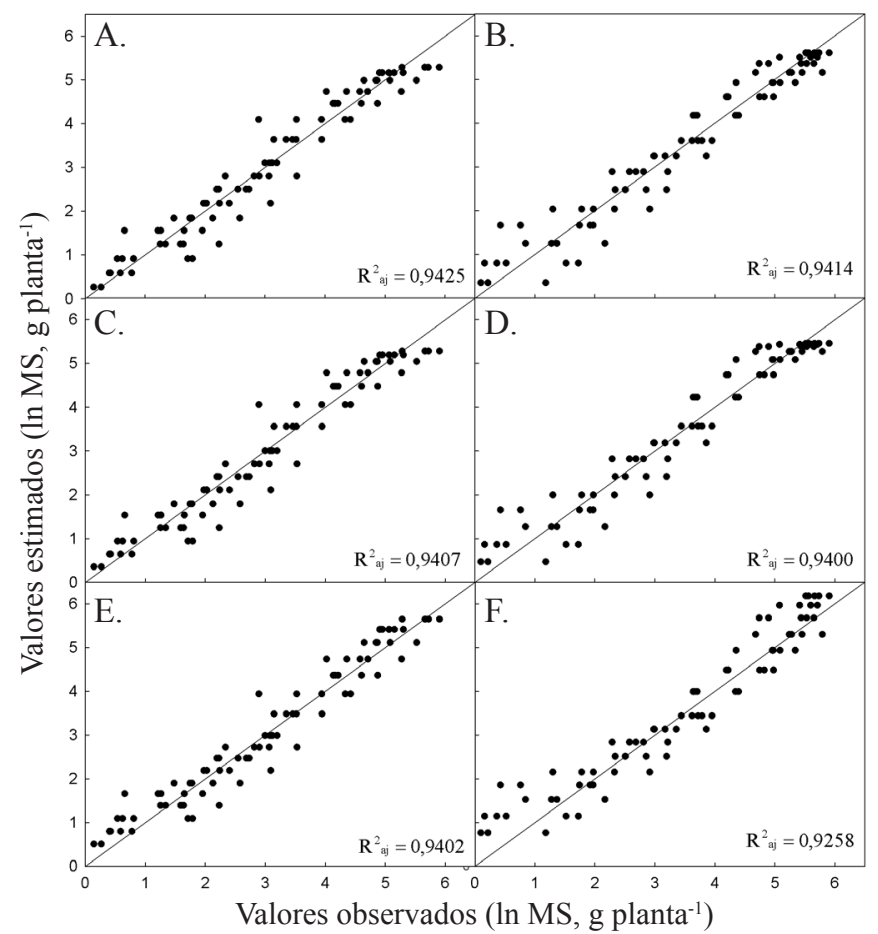

Figura 2. Valores de matéria seca acumulada (MS) observada e estimada pelos modelos expolinear (A e B), logístico (C e D) e Gompertz (E e F) para as cultivares de cana-de-açúcar RB92579 (A, C e E) e RB867515 (B, D e F) tendo graus-dia acumulados após o plantio como variável independente

et al. (2008) relatam ajuste satisfatório do modelo logístico aos dados de matéria seca da parte área (folhas, caule e espigas) com valores de $\mathrm{R}^{2}$ variando de 0,86 a 0,97 .

Andrade Neto et al. (2010) obtiveram, avaliando os efeitos de diferentes adubos verdes sobre o crescimento e produtividade da cultivar de sorgo BR 601, ajuste satisfatório deste modelo aos valores de matéria seca e fresca da parte aérea e altura das plantas cujos valores de coeficiente de determinação estiveram compreendidos entre 0,80 a $0,94,0,91$ a 0,99 e 0,91 a 0,99 , respectivamente para as variáveis massa fresca da parte aérea, massa seca da parte aérea e altura da planta. Sepaskhah et al. (2011) utilizaram indicadores de precisão para avaliar a capacidade do modelo logístico em simular o acúmulo de matéria seca e o rendimento de grãos do milho sob diferentes condições de suprimento de água e nitrogênio. Os autores concluíram que o modelo apresentou resultados aceitáveis, em termos de exatidão.

Similarmente aos demais modelos, o proposto por Gompertz forneceu estimativas satisfatórias do crescimento das cultivares de cana-de-açúcar, apresentando valores de $\mathrm{R}^{2}$ que variaram de 0,9247 a 0,9402 para as cultivares RB928064 e RB92579, respectivamente. Os mencionados valores estão em conformidade com os encontrados por Simões et al. (2005) quando ajustaram este modelo aos dados de biomassa total e produtividade da cana-de-açúcar para a região de Araras, São Paulo. Moura et al. (2008) avaliaram o ajuste dos modelos de crescimento expolinear, logístico e Gompertz ao acúmulo de matéria seca do milho e feijão-caupi em dois sistemas de cultivo. Segundo os autores, os melhores resultados foram apresentados pelo modelo de Gompertz.

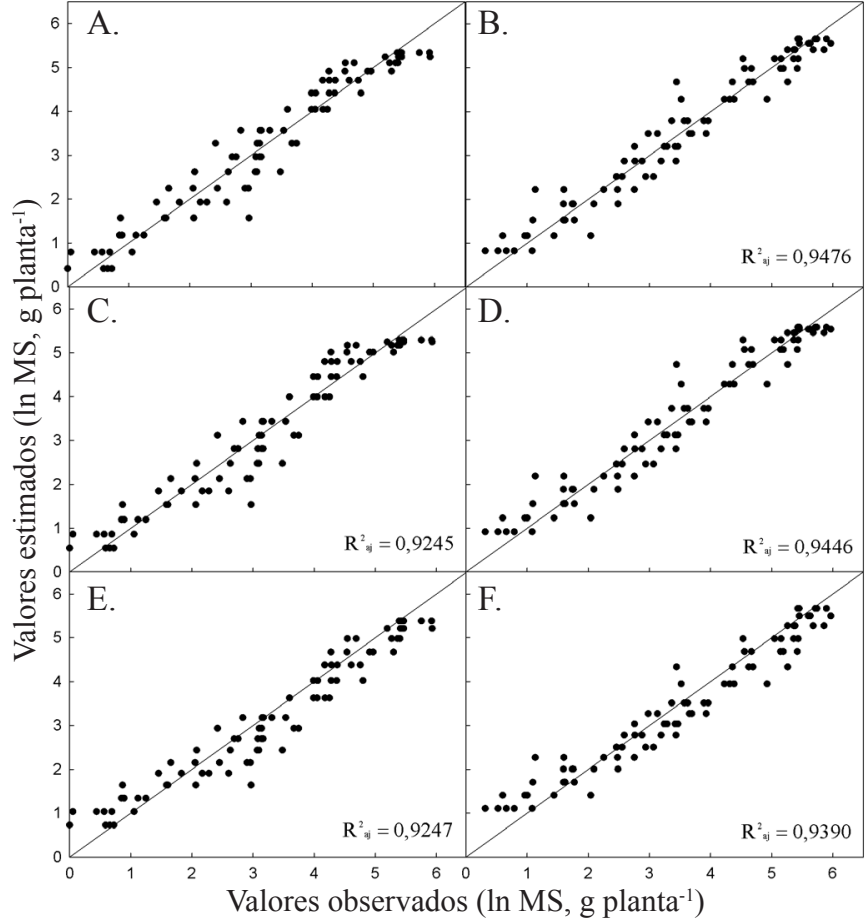

Figura 3. Valores de matéria seca acumulada (MS) observada e estimada pelos modelos expolinear (A e B), logístico (C e D) e Gompertz (E e F) para as cultivares de cana-de-açúcar RB928064 (A, C e E) e RB855453 (B, D e F) tendo graus-dia acumulados após o plantio como variável independente

Analisando as Figuras 2 e 3, letras A, B, C e D, verifica-se que, em geral, os valores estimados pelos modelos expolinear e logístico estiveram mais próximos da linha 1:1 no início do ciclo da cultura, indicando, assim, que houve maior proximidade dos valores estimados por esses modelos em relação aos valores observados; em contrapartida, constatou-se que o modelo de Gompertz apresentou maior tendência em superestimar os valores iniciais de matéria seca em relação aos demais modelos. Este mesmo padrão foi observado por Tei et al. (1996), ajustando este modelo ao acúmulo de massa seca da alface. Por outro lado, Simões et al. (2005) afirmam que este modelo apresentou boas estimativas do acúmulo de matéria seca da planta, sobretudo no início e no fim do ciclo da cana-de-açúcar.

$\mathrm{Na}$ Tabela 2 são apresentados os valores ajustados dos parâmetros dos modelos expolinear $\left(\mathrm{r}, \mathrm{c}_{\mathrm{m}} \mathrm{et}_{\mathrm{b}}\right)$, logístico $\left(\mathrm{r}_{\mathrm{e}} \mathrm{w}_{\mathrm{f}}\right)$ e Gompertz $\left(r\right.$ e $\left.r_{d}\right)$. Verificou-se significância estatística pelo teste $\mathrm{t}(\mathrm{p}<0,01)$ para todos os modelos e cultivares avaliadas. Pode-se observar que, independente da cultivar, os valores da taxa máxima de crescimento relativo (r), estimados pelo modelo de Gompertz, foram maiores que os dos modelos expolinear e logístico, sendo da ordem de 8 e $19 \%$, respectivamente. Tendência similar foi relatada por Tei et al. (1996) que afirmam que este modelo superestimou os valores de $\mathrm{r}$ em $40 \%$ para a cultura da alface. Lyra et al. (2003) verificaram, ao comparar os valores de $r$ estimados pelo modelo de Gompertz com os valores observados, superestimativas da ordem de $50 \%$ deste parâmetro. Os autores ressaltam que, do ponto de vista biológico, o parâmetro r deve ser interpretado com cautela quando estimado por este modelo. 
Tabela 2. Valores ajustados dos parâmetros dos modelos de crescimento expolinear, logístico e Gompertz para as cultivares de cana-de-açúcar RB92579, RB867515, RB928064 e RB855453*

\begin{tabular}{|c|c|c|c|c|c|}
\hline \multirow{2}{*}{ Modelo } & \multirow{2}{*}{ Parâmetro } & \multicolumn{4}{|c|}{ Cultivar } \\
\hline & & RB92579 & RB867515 & RB928064 & RB855453 \\
\hline \multirow{3}{*}{ Expolinear } & $r$ & $0,00400\left( \pm 2,7 \times 10^{-4}\right)$ & $0,00544\left( \pm 4,1 \times 10^{-4}\right)$ & $0,00487\left( \pm 3,9 \times 10^{-4}\right)$ & $0,00441\left( \pm 3,0 \times 10^{-4}\right)$ \\
\hline & $\mathrm{C}_{\mathrm{m}}$ & $0,219( \pm 0,043)$ & $0,244( \pm 0,036)$ & $0,177( \pm 0,026)$ & $0,262( \pm 0,038)$ \\
\hline & $t_{b}$ & $1395( \pm 103)$ & $1180( \pm 67)$ & $1122( \pm 77)$ & $1199( \pm 77)$ \\
\hline \multirow{2}{*}{ Logístico } & $r$ & $0,00361\left( \pm 1,9 \times 10^{-4}\right)$ & $0,00476\left( \pm 2,6 \times 10^{-4}\right)$ & $0,00400\left( \pm 2,4 \times 10^{-4}\right)$ & $0,00389\left( \pm 1,9 \times 10^{-4}\right)$ \\
\hline & $w_{f}$ & $243( \pm 46)$ & $242( \pm 29)$ & $215( \pm 31)$ & $287( \pm 35)$ \\
\hline \multirow{2}{*}{ Gompertz } & $\mathrm{R}$ & $0,00417\left( \pm 1,5 \times 10^{-4}\right)$ & $0,00608\left( \pm 2,1 \times 10^{-4}\right)$ & $0,00490\left( \pm 1,8 \times 10^{-4}\right)$ & $0,00476\left( \pm 1,6 \times 10^{-4}\right)$ \\
\hline & $r_{d}$ & $30,8 \times 10^{-5}\left( \pm 4,5 \times 10^{-5}\right)$ & $53,2 \times 10^{-5}\left( \pm 5,0 \times 10^{-5}\right)$ & $45,8 \times 10^{-5}\left( \pm 5,1 \times 10^{-5}\right)$ & $45,3 \times 10^{-5}\left( \pm 4,6 \times 10^{-5}\right)$ \\
\hline
\end{tabular}

* 0 erro padrão de estimativa dos parâmetros é apresentado entre parênteses

Dentre os modelos estudados, o logístico foi o que estimou as menores taxas máximas de crescimento relativo (r) com valores que variaram de 0,00361 a $0,00476 \mathrm{~g} \mathrm{~g}^{-1}{ }^{\circ} \mathrm{C}^{-1}$, para as cultivares RB92579 e RB867515, respectivamente. Lyra et al. (2003) observaram que os valores do parâmetro $r$ estimados por este modelo foram os que mais se aproximaram dos valores observados para três cultivares de alface, em comparação com os estimados pelos modelos expolinear e Gompertz. Na cultura do milho, este modelo foi ajustado aos dados de matéria seca da parte aérea por Lyra et al. (2008), que observaram valores de taxa máxima de crescimento relativo variando de 0,0034 a $0,0107 \mathrm{~g} \mathrm{~g}^{-1}{ }^{\circ} \mathrm{C}^{-1}$ e 0,0516 a $0,1712 \mathrm{~g} \mathrm{~g}^{-1} \mathrm{~d}^{-1}$, utilizando, respectivamente, as variáveis independentes graus-dia e dias após o plantio.

Dentre as cultivares estudadas, o maior valor de $\mathrm{r}$ foi apresentado pela RB867515, independente do modelo, o que leva a concluir que esta cultivar respondeu melhor às condições ambientais em que o estudo foi conduzido. Em contrapartida, os menores valores deste parâmetro foram observados na cultivar RB92579. A referida cultivar é a mais difundida no nordeste do Brasil cujos diversos estudos têm demonstrado sua superioridade em relação a outras cultivares (Almeida et al., 2008; Silva et al., 2011; Oliveira et al., 2010).

Com o propósito de visualizar o padrão de variação do acúmulo de matéria seca ao longo do período de formação da cana-de-açúcar (Figura 4), os valores ajustados dos parâmetros $\mathrm{c}_{\mathrm{m}}, \mathrm{r}$ e $\mathrm{t}_{\mathrm{b}}$ foram substituídos no modelo expolinear para as quatro cultivares avaliadas; no entanto, não foi realizada a linearização dos valores estimados por meio da aplicação do logaritmo, como apresentado nas Figuras 2 e 3, caso em que a matéria seca acumulada da parte aérea pode ser estimada pela Eq. 5:

$$
\mathrm{MS}=\left(\mathrm{c}_{\mathrm{m}} / \mathrm{r}\right) \ln \left(1+\exp \left(\mathrm{r}\left(\mathrm{GD}-\mathrm{t}_{\mathrm{b}}\right)\right)\right)
$$

Concomitantemente e com base nos valores de matéria seca da parte aérea estimados pelo modelo, obteve-se a taxa de crescimento instantânea da cultura (Figura 5) por meio da Eq. 6:

$$
\mathrm{TCC}=\left(\mathrm{MS}_{2}-\mathrm{MS}_{1}\right) /\left(\mathrm{GD}_{2}-\mathrm{GD}_{1}\right)
$$

em que:

TCC - taxa de crescimento da cultura, $\mathrm{g}$ planta ${ }^{-1} \mathrm{C}^{-1}$

$\mathrm{MS}_{2}$ - massa seca estimada pelo modelo no tempo térmico $\mathrm{GD}_{2}$, g planta $^{-1}$

$\mathrm{MS}_{1}$ - massa seca estimada pelo modelo no tempo térmico $\mathrm{GD}_{1}$, g planta ${ }^{-1}$

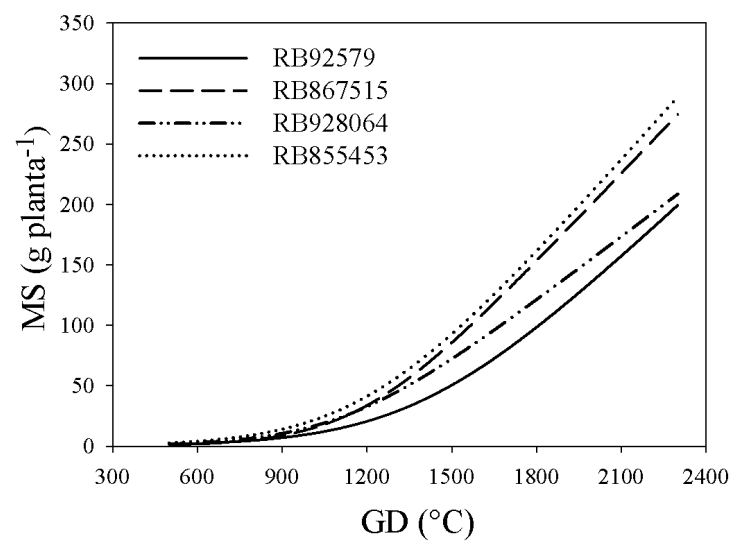

Figura 4. Massa seca acumulada da parte aérea (MS) de cultivares de cana-de-açúcar, estimada pelo modelo expolinear utilizando-se, como variável independente, graus-dia acumulados após o plantio (GD)

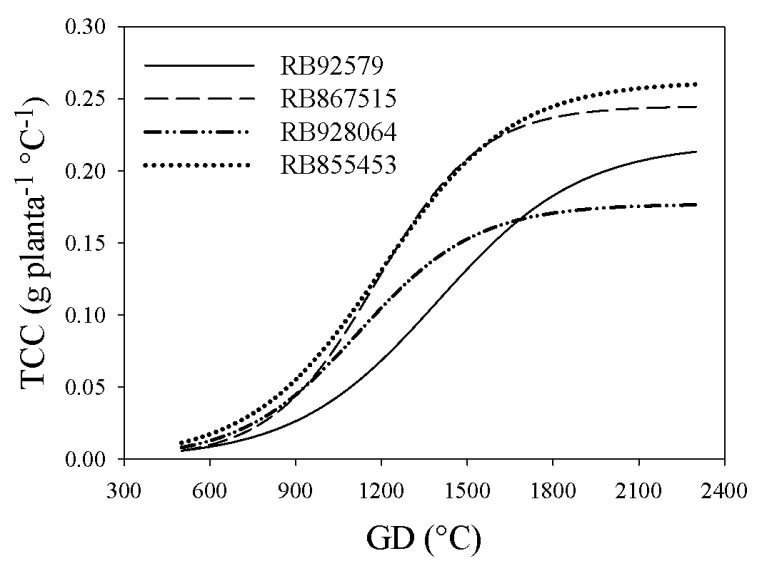

Figura 5. Taxa de crescimento da cultura (TCC) de cultivares de cana-de-açúcar em função dos graus-dia acumulados após o plantio (GD)

$$
\left(\mathrm{GD}_{2}-\mathrm{GD}_{1}\right) \text { - intervalo de graus-dia acumulado, }{ }^{\circ} \mathrm{C}
$$

Os resultados fornecidos pelas Eqs. 5 e 6 são expressos em g planta ${ }^{-1}$ e g planta ${ }^{-1} \mathrm{C}^{-1}$, respectivamente. Para que os valores de MS e TCC sejam expressos em unidades de área, basta utilizar o perfilhamento médio de cada cultivar ao final do período de formação da cultura. No presente experimento, aos $2200{ }^{\circ} \mathrm{C}$, os valores encontrados foram 11,5 $( \pm 0,7), 10,5( \pm 0,7), 12,8$ $( \pm 0,4)$ e $12,7( \pm 0,6)$ para as cultivares RB92579, RB867515, RB928064 e RB855453, respectivamente. O número de perfilhos observado no presente estudo esteve próximo dos 
relatados por Oliveira et al. (2010) ao estudarem o crescimento de cultivares de cana-de-açúcar sob irrigação plena.

$\mathrm{Na}$ simulação das curvas da taxa de crescimento, foi adotado o intervalo de $0,1{ }^{\circ} \mathrm{C}$, uma vez que intervalos menores também produziram o mesmo resultado matemático quando foi realizada a integração posterior das taxas de crescimento para obtenção do valor de massa seca acumulada para cada cultivar. Foi constatado que as curvas ajustadas pelo modelo expolinear para a massa seca acumulada da parte aérea foram bastante similares entre as cultivares até por volta dos $800^{\circ} \mathrm{C}$ (75 DAP) (Figura 4). Analisando a Figura 5, é possível verificar que a TCC das cultivares neste período foi baixa, com valores inferiores a $0,025 \mathrm{~g}$ planta $^{-1}{ }^{\circ} \mathrm{C}^{-1}$, o que caracterizou um período de incremento reduzido de biomassa, sendo observados valores compreendidos entre 12,2 e 20,5 g planta $^{-1}$ (Figura 4).

A partir dos $800{ }^{\circ} \mathrm{C}$, verificou-se aumento substancial na TCC, que se manteve crescente até o fim do experimento (205 DAP) quando as cultivares RB867515 e RB855453 se destacaram em relação às demais, apresentando valores de TCC da ordem de 15 e $30 \%$ superiores aos das cultivares RB92579 e RB928064, respectivamente.

Oliveira et al. (2004) observaram, estudando o crescimento de cultivares de cana-de-açúcar no Estado do Paraná, valores máximos de TCC da ordem de $11,0 \mathrm{~g} \mathrm{~m}^{-2} \mathrm{~d}^{-1}$, ocorridos até os 182 DAP. Farias et al. (2008) verificaram, avaliando os índices de crescimento da cana-de-açúcar sob diferentes condições de disponibilidade hídrica, que o ponto de máximo acúmulo de fitomassa seca da planta foi atingido aos 220 DAP cujo valor de TCC foi $0,7169 \mathrm{~g} \mathrm{~d}^{-1}$. Gava et al. (2001) constataram, estudando o crescimento e o acúmulo de nitrogênio de cultivares de canade-açúcar para as condições de Piracicaba, São Paulo, três fases distintas para o acúmulo de matéria seca da parte aérea, tendo a primeira ocorrido até os $60 \mathrm{DAP}$, caracterizada por crescimento lento; a segunda ocorreu dos 60 aos $210 \mathrm{DAP}$, quando foram observadas elevadas taxas de crescimento e, por fim, uma terceira fase, caracterizada como fase de maturação e pequeno acúmulo de fitomassa.

Ao final do período experimental (205 DAP), a cultivar RB928064 apresentou os menores valores de TCC $(0,18$ g planta $\left.{ }^{-1}{ }^{\circ} \mathrm{C}^{-1}\right)$ seguida, em ordem crescente, pela RB92579 $\left(0,21\right.$ g planta $\left.^{-1}{ }^{\circ} \mathrm{C}^{-1}\right), \operatorname{RB} 867515\left(0,24\right.$ g planta $\left.^{-1}{ }^{\circ} \mathrm{C}^{-1}\right)$ e RB855453 $\left(0,26\right.$ g planta $\left.^{-1}{ }^{\circ} \mathrm{C}^{-1}\right)$. Neste período a massa seca da cultivar RB855453 foi ligeiramente maior em comparação com a RB867515, as quais se destacaram quanto às cultivares RB928064 e RB92579.

\section{CONCLusões}

1. Os modelos expolinear, logístico e Gompertz estimaram, de forma satisfatória, a variação do acúmulo de matéria seca da parte aérea para as cultivares de cana-de-açúcar RB92579, RB867515, RB928064 e RB855453, durante a fase de formação da cultura.

2. Dentre os modelos avaliados, o que teve o melhor desempenho para o período estudado foi o expolinear, cujos valores do coeficiente de determinação ajustados foram superiores aos apresentados pelos demais modelos.
3. O modelo de Gompertz apresentou maior tendência em superestimar os valores iniciais de massa seca acumulada pelas cultivares.

4. Nas condições em que foi conduzido o experimento, as cultivares RB855453 e RB867515 apresentaram melhores respostas às condições de temperatura ambiente, uma vez que mantiveram o crescimento a taxas mais elevadas que as demais, o que resultou em maiores valores acumulados de massa seca, ao final do período de estudo.

\section{AgRAdECIMENTOS}

Esta pesquisa foi realizada com o apoio financeiro da Fundação de Amparo à Pesquisa do Estado de Minas Gerais (FAPEMIG) e do Conselho Nacional de Desenvolvimento Científico e Tecnológico (CNPq). O primeiro autor também gostaria de agradecer à Universidade Federal de Viçosa, por meio do Programa de Pós-Graduação em Meteorologia Agrícola, pela oportunidade para obtenção do título de Mestre.

\section{Literatura Citada}

Almeida, A. C. dos S.; Souza, J. L.; Teodoro, I.; Barbosa, G. V. S.; Moura Filho, G.; Ferreira Júnior, R. A. Desenvolvimento vegetativo e produção de variedades de cana-de-açúcar em relação à disponibilidade hídrica e unidades térmicas. Ciência e Agrotecnologia, v.32, p.1441-1448, 2008.

Amer, F. A.; Williams, W. T. Leaf-area growth in Pelargonium zonale. Annals of Botany, v.21, p.339-342, 1957.

Andrade Neto, R. C.; Miranda, N. O.; Duda, G. P.; Góes, G. B.; Lima, A. S. Crescimento e produtividade do sorgo forrageiro BR 601 sob adubação verde. Revista Brasileira de Engenharia Agrícola e Ambiental, v.14, p.124-130, 2010.

Benincasa, M. M. P. Análise de crescimento de plantas: Noções básicas. Jaboticabal: FUNEP, 1988. 42p.

CONAB - Companhia Nacional de Abastecimento. Cana-deaçúcar: Safra 2011/2012 - terceiro levantamento. http:// www.conab.gov.br. 13 Set. 2012.

Confalone, A. E.; Bernardes, M. S.; Costa, L. C.; Righi, C. A.; Dourado Neto, D.; Martin, T. N.; Manfron, P. A.; Pereira, C. R. Expolinear model on soybean growth in Argentina and Brazil. Ciência Rural, v.40, p.1009-1016, 2010.

Costa, L. C.; Ferreira, E. A.; Sediyama, G. C.; Mantovani, E. C.; Cecon, P. R. Uso da função expolinear para análise do crescimento da cultura da soja em diferentes condições de disponibilidade de água no solo. Revista Brasileira de Agrometeorologia, v.7, p.1-4, 1999.

Farias, C. H. de A.; Fernandes, P. D.; Azevedo, H. M. de; Dantas Neto, J. Índices de crescimento da cana-de-açúcar irrigada e de sequeiro no estado da Paraíba. Revista Brasileira deEngenharia Agrícola e Ambiental, v.12, p.356-362, 2008.

Gascho, G. J.;Shih, S. F.; Sugarcane. In: Teare, I. D.; Peet, M. M. (ed.). Crop-Waterrelations. New York: Wiley-Interscience, 1983. p.445-479.

Gava, G. J. de C.; Trivelin, P. C. O.; Oliveira, M. W. de; Penatti, C. P. Crescimento e acúmulo de nitrogênio em cana-deaçúcar cultivada em solo coberto com palhada. Pesquisa Agropecuária Brasileira, v.36, p.1347-1354, 2001. 
Goudriaan, J.; Monteith, J. L. A mathematical function for crop growth based on light interception and leaf area expansion. Annals of Botany, v.66, p.695-701, 1990.

Karadavut, U.; Palta, Ç.; Kökten, K.; Bakoglu, A. Comparative study on some non-linear growth models for describing leaf growth of maize. International Journal of Agriculture \& Biology, v.12, p.227-230, 2010.

Lyra, G. B.; Souza, J. L. de; Lyra, G. B.; Teodoro, I.; Moura Filho, G. Modelo de crescimento logístico e exponencial para o milho BR 106, em três épocas de plantio. Revista Brasileira de Milho e Sorgo, v.7, p.211-230, 2008.

Lyra, G. B.; Zolnier, S.; Costa, L. C.; Sediyama, G. C.; Sediyama, M. A. N. Modelos de crescimento para alface (Lactuca sativa L.) cultivada em sistema hidropônico sob condições de casa-de-vegetação. Revista Brasileira de Agrometeorologia, v.11, p.69-77, 2003.

MAPA - Ministério da Agricultura, Pecuária e Abastecimento. Projeções do agronegócio 2009/2010 a 2019/2020. http:// www.agricultura.gov.br/vegetal/culturas/ cana-de-açúcar. 13 Set. 2012.

Moura, M. S. B. de; Souza, L. S. B. de; Silva, T. G. F. da; Soares, J. M.; Carmo, J. F. A. do; Brandão, E. O. Modelos de crescimento para o feijão-caupi e o milho, sob sistemas de plantio exclusivo e consorciado, no semiárido brasileiro. Revista Brasileira de Agrometeorologia, v.16, p.275-284, 2008.

Oliveira, E. C. A. de; Oliveira, R. I. de; Andrade, B. M. T. de; Freire, F. J.; Lira Júnior, M. A.; Machado, P. R. Crescimento e acúmulo de matéria seca em variedades de cana-deaçúcar cultivadas sob irrigação plena. Revista Brasileira de Engenharia Agrícola e Ambiental, v.14, p.951-960, 2010.

Oliveira, R. A.; Daros, E.;Zambon, J. L. C.; Weber, H.; Ido, O. T.; Zuffellato-Ribas, K. C.; Koehler, H. S.; Silva, D. K. T. Crescimento e desenvolvimento de três cultivares de canade-açúcar, em cana-planta, no estado do Paraná. Scientia Agraria, v.5, p.87-94, 2004.
Ribeiro,A. C.; Guimarães, P. T. G.;Alvarez V., V.H. Recomendações para o uso de corretivos e fertilizantes em Minas Gerais. 5. ${ }^{\mathrm{a}}$ aproximação. Viçosa: CFSEMG, 1999. 322p.

Richards, F. J. A flexible growth function for empirical use. Journal of Experimental Botany, v.10, p.290-300, 1959.

Santos, V. R. dos; Filho, G. M.; Albuquerque, A. W. de; Costa, J. P. V. da; Santos, C. G. dos; Santos, A. C. I. dos. Crescimento e produtividade agrícola de cana-de-acúcar em diferentes fontes de fósforo. Revista Brasileira de Engenharia Agrícola e Ambiental, v.13, p.389-396, 2009.

Sepaskhah, A. R.; Fahandezh-Saadi, S.; Zand-Parsa, S. Logistic model application for prediction of maize yield under water and nitrogen management. Agricultural Water Management, v.99, p.51-57, 2011.

Silva, T. G. F. da; Moura, M. S. B. de; Zolnier, S.; Carmo, J. F. A. do; Souza, L. S. B. de. Biometria da parte aérea da cana soca irrigada no submédio do vale do São Francisco. Revista Ciência Agronômica, v.43, p.500-509, 2012.

Silva, T. G. F. da; Moura, M. S. B. de; Zolnier, S.; Soares, J. M.; Vieira, V. J. de S.; Gomes Júnior, W. F. Demanda hídrica e eficiência do uso de água da cana-de-açúcar irrigada no semiárido brasileiro. Revista Brasileira de Engenharia Agrícola e Ambiental, v.15, p.1257-1265, 2011.

Simões, M. dos S.; Rocha, J. V.; Lamparelli, R. A. C. Growth indices and productivity in sugarcane.ScientiaAgrícola, v.62, p.23-30, 2005.

Smit, M. A.; Singels, A. The response of sugarcane canopy development to water stress. Field CropsResearch, v.98, p.91-97, 2006.

Sousa, E. L. de; Macedo, I. de C. Etanol e bioeletricidade: A cana-de-açúcar no futuro da matriz energética. São Paulo: Luc Projetos de Comunicação, 2010. 315p.

Tei, F.; Aikman, D. P.; Scaife, A. Growth of lettuce, onion na dred beet. 2. Growth modelling. Annals of Botany, v.78, p.645-652, 1996. 